\title{
The EnMAP Mission Planning System
}

\author{
Thomas Fruth*, Christoph Lenzen ${ }^{\dagger}$, Elke Gross ${ }^{\ddagger}$ and Falk Mrowka ${ }^{\S}$ \\ Deutsches Zentrum für Luft- und Raumfahrt e. V., German Aerospace Center \\ Münchener Straße 20, 82234 Weßling, Germany
}

\begin{abstract}
The Environmental Monitoring and Analysis Program mission (EnMAP) is a German hyperspectral Earth observation mission, currently scheduled for launch in 2020. The EnMAP Mission Planning System (MPS), developed and operated by the German Space Operations Center (GSOC), is one of 15 subsystems constituting the EnMAP Ground Segment. Its main task is to compile and maintain a conflict-free timeline for routine operations that does not violate any constraints of the spacecraft (e.g. regarding power or onboard memory); this timeline will regularly be commanded to the spacecraft.

This paper gives an overview of the current EnMAP MPS design, including the special requirements of the EnMAP mission, the components of the MPS and its most important external interfaces. The design of the EnMAP MPS largely builds on our experience gathered during the TerraSAR-X/TanDEM-X mission and has been developed further. Novel technologies include the Reactive Planning framework, which particularly stands out due to its high responsiveness to user input. Particular attention is furthermore paid to the inclusion of cloud coverage and sunglint information into the planning process-two challenges which are specific to EnMAP observing in the optical and near infrared part of the spectrum.
\end{abstract}

\section{Introduction}

\section{A. EnMAP Mission}

Understanding the state and evolution of terrestrial and aquatic ecosystems requires an accurate quantitative data base. Earth observations using imaging spectroscopy yield spatially resolved reflectance spectra and may thus provide a crucial resource for studying vital processes on the Earth's surface: Important scientific applications include studies of the vegetation (e. g. regarding its type or environmental stresses) [1], coastal and inland waters (e. g. regarding water quality or constituents) [2], the soil (e. g. for mapping or regarding erosion) [3], geology (e. g. for exploration) [4], snow and ice (e.g. to improve snowmelt models) [5], or urban studies (e. g. regarding development, planning, or risk assessment) [6].

The Environmental Monitoring and Analysis Program mission (EnMAP) [7] is a German hyperspectral Earth observation mission currently scheduled for launch in 2020. Located in in a sun-synchronous polar orbit with a local time of $11 \mathrm{~h}$ (descending node), it will provide a global revisit capability of four days (within an off-nadir range of $\pm 30^{\circ}$ ) which cannot be achieved with conventional airborne sensors; one full repeat cycle lasts 27 days (398 revolutions). Its dual-spectrometer instrument [8] covers an optical spectral range between 420 and $2450 \mathrm{~nm}$ with a spectral sampling distance of 4.8-12 $\mathrm{nm}$ and a ground sampling of $30 \mathrm{~m}$, respectively. Together with a high signal-to-noise ratio, these optical characteristics will enable environmental studies as described above with an unprecedented level of detail w.r.t. current spaceborne sensors.

A full description of the EnMAP mission is given by [7], whereas the EnMAP science plan [9] addresses the mission's scientific applications in detail.

\section{B. EnMAP Project Structure}

The overall project management of the EnMAP mission is led by the Space Administration of the German Aerospace Center (DLR). The project is structured into three segments: First, the user segment coordinates the mission's objectives [9] as well as the scientific exploitation of the data; it is led by the German Research Center for Geosciences (GFZ) as

\footnotetext{
*Mission Planning System Engineer, Mission Operations department, German Space Operations Center, Thomas.Fruth@ dlr.de

${ }^{\dagger}$ Mission Planning System Engineer, Mission Operations department, German Space Operations Center, Christoph.Lenzen@dlr.de

$\doteqdot$ Mission Planning System Engineer, Mission Operations department, German Space Operations Center, Elke.Gross@ dlr.de

${ }^{\S}$ Head of Mission Planning Team, Mission Operations department, German Space Operations Center, Falk.Mrowka@dlr.de
} 
the mission principal scientific investigator. Second, the space segment is responsible for producing the bus and the hyperspectral imager, its integration and testing, as well as the launch; these activities are led by OHB System AG. Third, the ground segment [10-12] links the user and space segment by providing facilities and systems for operating the satellite and retrieval of the targeted scientific data; it is led by the Earth Observation Center (EOC) and the German Space Operations Center (GSOC) at DLR.

The EnMAP ground segment is divided further into three main systems: First, the Mission Operations System (MOS) is responsible for overall operations and monitoring of both the satellite and its payload; it is established by GSOC, which hosts the control room and operator team in a multi-mission environment. Second, the Processor, Calibration, and Validation System (PCV) is responsible for developing the hyperspectral processing chain for radiometrically calibrated, spectrally characterized and geometrically corrected imaging products [11,13]; this system is coordinated by the Remote Sensing Technology Institute (IMF) at DLR. Third, the Payload Ground System (PGS) is responsible for data reception, processing and archiving, as well as to establish a web interface to the scientific user community [14]; it is established by the German Remote Sensing Data Center (DFD) at DLR.

The three ground segment systems are finally broken down into 15 subsystems; their products, mutual interaction as well as the tracing of requirements and tests are described in a detailed development model [15]. Integration and Technical Verification and Validation (ITVV) activities will demonstrate that all project specifications are fulfilled; for the ground segment, these are organized in eleven phases from subsystem to mission level [15].

\section{Mission Planning System}

The EnMAP Mission Planning System (MPS) is one of four subsystems within the EnMAP MOS; it is developed and operated by GSOC.

Main task of the EnMAP MPS is to compile and maintain a conflict-free timeline for routine operations (bus and payload) that does not violate any constraints of the spacecraft or on ground. For this purpose, the EnMAP MPS automatically ingests various types of inputs from other subsystems: Examples include Earth observation requests from scientific users, calibrations, orbit updates, orbit maneuver opportunities, and ground station availabilities. In order to evaluate the feasibility for scheduling individual activities, the MPS keeps a dynamic planning model covering spacecraft resources such as onboard power and memory as well the corresponding constraints. Furthermore, it implements the mission policy for prioritizing between competing requests in the case of conflicts.

The EnMAP MPS also informs other subsystems about timeline updates on a regular basis, for instance to provide the scientific user with the current execution status of an Earth observation request. However, the main MPS output product is the timeline itself, which will automatically and regularly be commanded to the spacecraft through the EnMAP Flight Operations System (FOS; also part of MOS).

\section{MPS Design}

The design of the EnMAP MPS largely builds on our experience gathered during the TerraSAR-X/TanDEM-X mission [16] and has been developed further. Parts of a preliminary EnMAP MPS design have already been described by [17]; however, it has also evolved significantly since then - most importantly through incorporating the most recent Mission Planning technologies in the context of an intermediate critical design review (so-called $\triangle \mathrm{CDR}$ ) of the EnMAP ground segment in 2016.

The following sub-sections focus on the current EnMAP design by describing its specifications (II.A), external interfaces (II.B), and internal static architecture (II.C).

\section{A. Requirements}

The specifications of the EnMAP MPS are detailed in a number of subsystem-specific requirements, which are briefly summarized in the following text.

First of all, the MPS shall be the central element for planning of routine EnMAP payload operations. This includes importing Acquisition Requests (AR; including normal Earth observations, calibrations, as well as background mission requests) from $24 \mathrm{~h}$ up to two weeks in advance, calculating the corresponding observation opportunities (using off-nadir pointing limits and the most recent orbit), rejecting unfeasible (e. g. unduly late) ARs, and regularly informing the user about the current planning status. Two additional requirements are very specific to EnMAP observing in the optical and near infrared spectrum: Mission Planning shall take cloud coverage and sunglint into account (see Sections III.B and III.C, respectively, for details). 
In order to generate an activity schedule of feasible ARs and other spacecraft activities, the MPS shall be capable of modeling the onboard battery power, mass memory, and time-tagged command buffer f each including its respective dynamic evolution. Further planning constraints are e. g. to separate certain time-tagged operations such that they do not overlap or to consider spacecraft swing times between consecutive image acquisitions. Finally, the planning process must be able to resolve any conflicts between data takes autonomously.

Further important activities that shall be coordinated by the MPS include ground station contacts and orbit maneuvers. For ground station contacts, this task includes computing contact times and coordinating availabilities with the respective station. For maneuvers, Mission Planning shall be able to ingest a list of alternative maneuver opportunities from Flight Dynamics, select and schedule an opportunity best fitting the current timeline, and update this selection as needed.

Finally, the MPS shall generate an activity schedule which can be used for generating and uploading the corresponding commands to the spacecraft.

\section{B. External Interfaces}

The most important external interface items of the EnMAP MPS are shown in Fig. 1 and described in the following.

- Flight Operations System (FOS) provides MPS with flight procedures, informs MPS about outage times that are not available for routine operations, and gives feedback on the success of command uplinks. In turn, MPS delivers its timeline to FOS for commanding both in the Launch and Early Orbit Phase (LEOP; so-called "background sequence") as well as during routine operations.

- Flight Dynamics System (FDS) provides MPS with updated orbit information regularly. To support commanding of observations with precise attitude steering, guidance profiles are returned on request. Furthermore, maneuver opportunities are announced by FDS (and then selected by MPS) through a dedicated interface.

- Ground Data Systems (GDS) inform MPS about S-band passes scheduled for command uplink.

- Neustrelitz Ground Station (NSG) informs MPS about X-band downlink opportunities, which may then be requested by MPS. Details regarding a commanded uplink are forwarded by MPS through the "Downlink Info", whereas NSG in turn provides a report after the data reception.

- Data and Information Management System (DIMS) provides all user input regarding nominal payload operations. Most importantly, this includes the submission of ARs, which contain all parameters required for scheduling nominal EnMAP image acquisitions (i. e. Earth observations and calibrations). In turn, MPS provides DIMS with regular status updates regarding any submitted AR.

- Instrument Planning provides the user with a website to prepare and submit scientific observations. MPS supports this functionality with a back end web service that provides a swath preview (and corresponding parameters, e.g. the off-nadir angle) for potential observations prior to the subsequent order workflow (through DIMS; see above) itself.

- External Auxiliary Data Sources provide MPS with cloud coverage forecasts and statistics.

\section{Static Architecture}

Figure 1 also gives a schematic overview of the EnMAP MPS architecture. Therein, the actual color of a specific Mission Planning item indicates its reusability from earlier missions or for upcoming missions: Green denotes generic items, which only need to be configured mission specifically, whereas blue items need to be adapted mission specifically; orange items are considered as EnMAP specific.

The following sections provide an overview of the four main logical components of the MPS: MPS Import Handler, MPS Core, MPS LEOP Tools, and MPS Cloud Coverage Handling; these are marked in Figure 1 with light gray boxes.

\section{MPS Import Handler}

The MPS Import Handler encompasses items that accept planning input from various other subsystems. It accepts EnMAP specific input, parses it into a generic Mission Planning format and then passes this to the MPS Core component. Input items are either ingested as files (File Ingestion) or through a dedicated web service handling ARs from DIMS (Acquisition Request Ingestion) and then transformed to Reactive Planning messages.

*The limited onboard buffer of 120 time-tagged commands is a rather unusual characteristic of the EnMAP mission; for a more detailed discussion in the context of the EnMAP MPS, see [17]. 


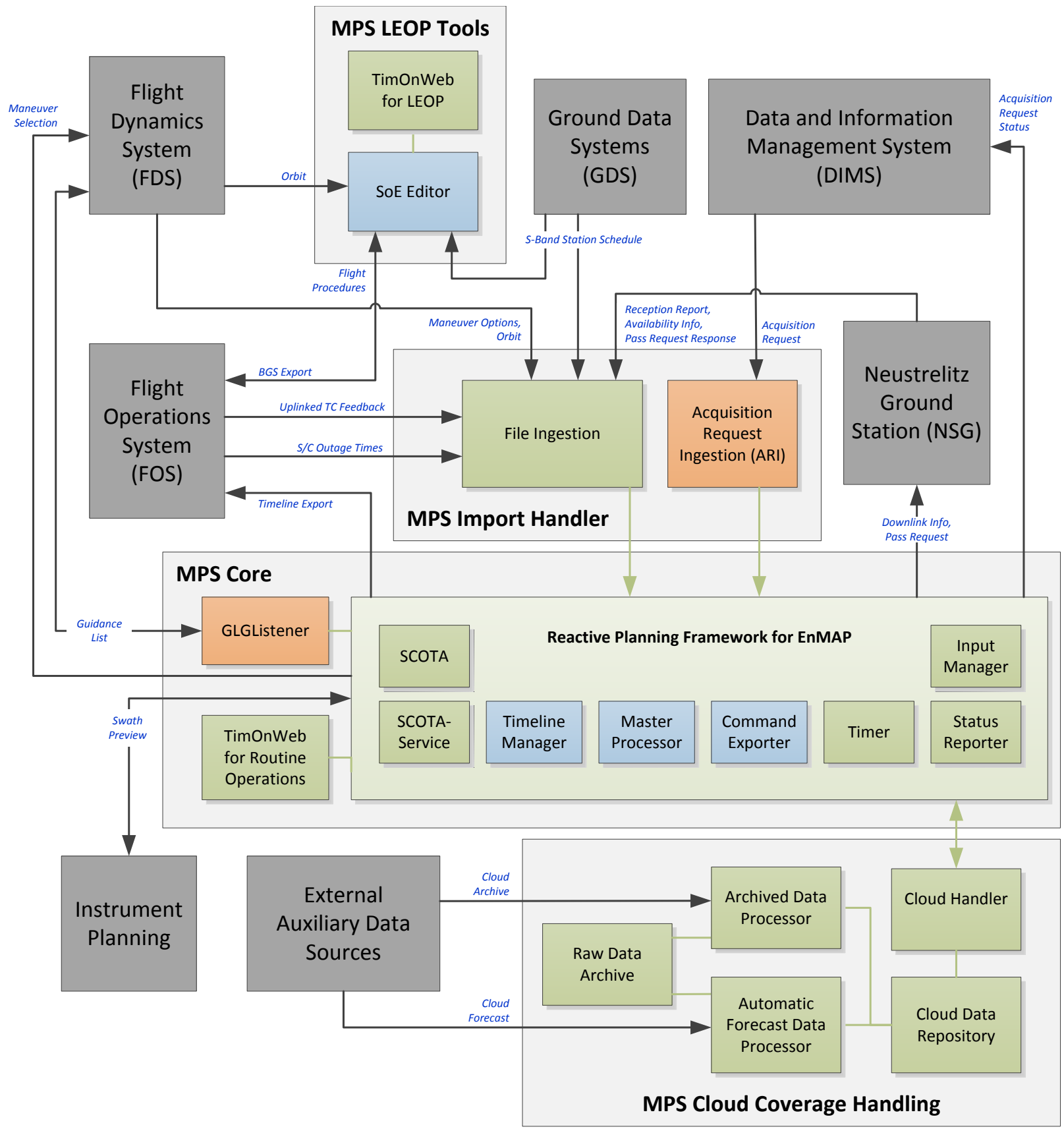

Fig. 1 EnMAP MPS Static Architecture and Interfaces

\section{MPS Core}

The MPS Core component forms the central part of the EnMAP planning system: Using the Reactive Planning Framework (see Section III.A with project-specific adaptations for EnMAP, MPS Core receives input via the MPS Import Handler, produces a conflict-free timeline, and forwards the relevant output to other subsystems (e. g. for commanding). Furthermore, the MPS Core component handles the guidance list generation and provides the swath preview functionality to Instrument Planning, a timeline preview to spacecraft operation engineers, and status updates to the sender of an order. 


\section{MPS LEOP Tools}

The MPS LEOP Tools component provides the means for building, editing, and visualizing the Sequence of Events (SoE) before and during LEOP. Furthermore, it enables the MPS operator to regularly export a "Background Sequence" of recurring basic commands during LEOP. Both configuration items that are used for this purpose, TimOnWeb and the SoE Editor, are generic MPS tools; these are described in detail in [18].

\section{MPS Cloud Coverage Handling}

This MPS component automatically ingests cloud coverage data (forecast and statistical information), processes the data and stores it into a database such that it is readily available for the Reactive Planning Framework for EnMAP on request. The component is specifically developed for EnMAP, but completely generic and may thus be reused for any future mission. More details regarding the dynamic cloud coverage handling within the EnMAP MPS can be found in Section

\section{Novel Mission Planning Concepts}

Compared to previous planning systems at GSOC, the EnMAP MPS will include a number of novel technologies. Most importantly, it will be the first system which makes use of the Reactive Planning framework and to include cloud coverage as well as sunglint information already during planning. These concepts are described in more detail in the following three sub-sections.

\section{A. Reactive Planning}

The central planning of the EnMAP planning system is based upon GSOC's new Reactive Planning framework (formerly called Incremental Planning System, see [19]), which has been designed to meet the customers' desires according to the experiences gathered in previous missions, especially the TerraSAR-X/TanDEM-X mission ([20], [21]).

\section{Objectives}

The main objectives within the EnMAP context are

1) immediate response about the planning states of all orders: as soon as new input is available (e.g. new order, new orbit information), a planning run is performed and changes in order status are communicated

2) late order deadline: no possibly long lasting planning process needs to be performed before an uplink, which allows accepting orders shortly before the uplink passage

3) robustness against IT-failures: all information received and all timeline updates calculated shall be persisted in a way that re-starting the system results in the state before the crash.

\section{Core Reactive Planning Components}

Reactive Planning has been designed as a set of cooperative components, which communicate via ActiveMQ messages. Persistence for both, ActiveMQ and components, is implemented based upon a Cassandra database. The Reactive Planning Framework for EnMAP consists of the following core Reactive Planning components:

1) InputManager: Collects all messages to the MasterProcessor and forwards them one by one. Allows prioritization in case of multiple pending messages and then even interrupting an ongoing calculation.

2) MasterProcessor: Maintains the planning model and the master timeline. Incorporates the messages from the InputManager into the model and timeline in a single-threaded way. Project-specific code is required in order to implement the desired behaviour of all possible input ingested through the MPS Import Handler (see Fig. 1 and Section II.B. . However, this will be based on generic modelling capabilities, scheduling algorithms and event calculation functionality.

3) TimelineManager and CommandExporter: Extract the timeline-to-command from the MasterProcessor's master timeline and derive the commands, which need to be sent to the spacecraft. The TimelineManager sends commands to FOS and receives feedback about uplink success. It creates a message about the difference of the commands on-board and the master timeline, which is sent to the MasterProcessor via the InputManager. Similar to the MasterProcessor, the TimelineManager requires mission-specific code, which implements the way to derive the timeline-to-command from the master timeline.

4) Timer: This is the only component with access to the current time. It sends configurable messages to the 
InputManager at configurable times, e. g. in order to trigger a command export. The timer is aware of the current model and timeline and therefore can react on newly ingested uplink station contacts. It supports real-time for operations and sim-time for tests and simulation.

\section{Extensions to Core Components}

In addition to the Reactive Planning core components, the EnMAP MPS includes some extensions to serve project-specific requirements:

1) StatusReporter: Has access to the current planning model state and provides DIMS with relevant updates regarding AR status changes (actively and on request).

2) SCOTA (SpaceCraft Orbit and groundTrack Analysis Tool) is a generic GSOC library that performs orbit-related calculations and analyses in the Mission Planning context. Within the EnMAP Reactive Planning framework, it is for instance invoked by the scheduling algorithms of the MasterProcessor to calculate potential acquisition times and observing geometries from incoming Acquisition Requests.

3) SCOTA-Service: Based upon the SCOTA library, this web service obtains preview requests from the EnMAP user through a front end provided by Instrument Planning. In addition to a preview of the ground swath, the response contains information regarding the observing geometry (e.g. off-nadir angle). The design of the final version furthermore envisages to include locally resolved sunglint information (III.C) as well as a most recent cloud forecast from the corresponding MPS service (III.B).

Since both the preview service and the final scheduling algorithms are provided by MPS (using the same algorithms and orbit products), the user benefits from a completely consistent ordering workflow.

\section{B. Cloud Data Handling and Order Prioritization}

EnMAP will perform Earth observations in the visible and near infrared (VNIR) as well as shortwave infrared (SWIR) part of the electromagnetic spectrum. Clouds in the acquired scenes are thus expected to reduce the area that is useful for scientific applications by more than 50\% [17, 22]. However, there are two potential counter measures against wasting satellite resources for largely obstructed observations: Onboard cloud detection to discard affected scenes before downlink (e. g. [23] in the context of EnMAP) or prioritizing acquisitions with favorable cloud forecasts already on-ground (e. g. in use for Landsat 7 [22] or CHRIS-PROBA [24]).

The EnMAP mission decided to implement the latter option, i. e. to take cloud coverage into account at Mission Planning [23]. Albeit largely driven by economic considerations, this yields another advantage: Some additional daytime passes over Europe may be used for downlink if the cloud forecast for competing image acquisitions is unfavorable. Furthermore, the user can receive status information regarding his order already prior to the acquisition and may react accordingly. On the other hand, on-ground models can of course never achieve the accuracy available through realtime in situ evaluation; instead, their benefit is largely determined by the precision of the underlying cloud data.

The following two sub-sections (III.B.1 and III.B.2) focus on the corresponding data sets with forecast and statistical cloud data, respectively, as well as their technical integration into the EnMAP MPS; finally, Section III.B.3 describes the process of prioritizing ARs using cloud data as well as other relevant input parameters.

\section{Statistical Cloud Data}

In the planning context, statistical cloud data can be useful for mission analysis (see e. g. [25]) or during routine operations for preferring scenes with above-average weather conditions (e.g. cloudless winter scene in Europe) with respect to areas with more frequent clear skies (e. g. deserts; see also [22] for a similar approach). Today the most comprehensive and consistent datasets of statistical cloud properties are provided by the Cloud_cci project within the Climate Change Initiative of the European Space Agency (ESA). From the six available Cloud_cci datasets [26], the EnMAP MPS uses the dataset covering the longest baseline (1982-2014): Daily cloud fraction probabilities in 10\% bins are based on the Cloud_cci L3U cloud mask data of the AVHRR-PM dataset [27]. In the baseline implementation, the system will use a geographical resolution of $0.25^{\circ}$ with nearest-neighbor interpolation for intermediate coordinates.

Given these histograms and the user cloud coverage threshold $C_{\text {limit }}$ (see Section III.B.3, the statistical probability that a scenario of actual cloud coverage below $C_{\text {limit }}$ is encountered can be calculated as $\xi_{H}\left(C_{\text {limit }}, \lambda, \varphi, t\right)$, whereby $(\lambda, \varphi)$ specifies the center coordinate and $t$ the acquisition time of a given data take, respectively. 


\section{Forecasted Cloud Data}

Cloud forecasts constitute the most important parameter for prioritizing ARs (see next Section [II.B.3). For the EnMAP GS, the German Meteorological Office (DWD) has been selected as the provider for cloud forecast data. Its current global weather prediction model ICON [28] is publicly available and contains the actual cloud coverage $C_{A}$ (in percent) as a direct output parameter. The model has a geographical resolution of approximately $13 \mathrm{~km}$; it runs four times daily and yields hourly sampled predictions within a forecast horizon of three days.

Previous experiments with cloud forecast data at GSOC have shown that a simple linear interpolation yields the most reliable results for arbitrary intermediate times [29]. Due to this and an improved sampling of the ICON model w.r.t. that earlier study ( $1 \mathrm{~h}$ instead of $6 \mathrm{~h}$ ), the baseline EnMAP MPS implementation will use a most simple nearest-neighbor interpolation.

For an ingested AR, each corresponding ground swath $S$ will be suitably sampled into a set of non-intersecting sub-tiles $S_{i}\left(S=\bigcup_{i} S_{i}\right)$ of constant $C_{A}\left(S_{i}, t\right)$, i. e. following the model grid. This can be used to calculate the expected relative cloud coverage of the whole swath, namely

$$
\left\langle C_{A}(S, t)\right\rangle=\frac{\sum_{i}\left[A\left(S_{i}\right) \cdot C_{A}\left(S_{i}, t\right)\right]}{\sum_{i} A\left(S_{i}\right)},
$$

whereby $A\left(S_{i}\right)$ describes the ground coverage area for each sub-tile $S_{i}$.

\section{Prioritization of Earth Observations}

Conflicts between data takes will be resolved in the MPS automatically. This involves a priority concept for ARs, which may lead to certain data takes not being executed. The MPS calculates a benefit $B$ for each AR and considers it for scheduling: When a new request is submitted, it is included into the timeline unless there is a conflict with an existing activity. In the case of a conflict, scheduled activities are tried to be removed from the timeline while obeying the rule that the new request may not affect any scheduled request of greater or equal benefit. Existing activities are removed in increasing order of benefit and number and within a certain timeline horizon (e.g. $\pm 12 \mathrm{~h}$ around the conflict). In case the conflicting request was planned successfully, the scheduling process tries to reschedule removed activities (in reverse order as for the removal).

For a request of execution time $t$ (mid-time of image acquisition), the benefit $B$ includes the following input parameters:

- From the AR:

- priority $p$ (with $p \in[1,2 \ldots 5]$, where 1 denotes the highest priority)

- user category (used to distinguish user types in addition to priority, e. g. institutional users)

- cloud coverage threshold $C_{\text {limit }}$ (max. allowed fraction of swath covered by clouds; alternatively, the user may configure $C_{\text {limit }}$ as a minimum threshold to explicitly target at clouds - however, this mode works likewise and is not described in the following for better readability)

- statistical probability $\xi_{H}\left(C_{\text {limit }}, \lambda, \varphi, t\right)$ (see Section III.B.1)

- actual cloud coverage $\left\langle C_{A}(S, t)\right\rangle$ (see Section III.B.2)

These parameters are combined into a set of functions $\left\{b_{i}\right\}$, which are then weighted to calculate a single benefit value

$$
B=\sum_{i} w_{i} b_{i}
$$

In its initial configuration, the EnMAP MPS will use a hierarchical weighting (i.e. $w_{1} \gg w_{2} \gg \ldots$ ) with the following main characteristics:

1) Presumably cloud-free acquisitions (i. e. with $\left\langle C_{A}(S, t)\right\rangle \leq C_{\text {limit }}$ ) yield a higher benefit than any acquisition with an unfavorable forecast, regardless of the user priority or any other parameter. In order to obtain a stable planning process for the scientific users, cloud forecasts will enter the benefit calculation once the data is sufficiently reliable (e. g. $48 \mathrm{~h}$ in advance) and not be updated as new forecast data may become available.

2) Within each group of acquisitions (good/bad cloud forecast), benefit values are strictly ordered by priority (e. g. $p=1$ yielding $b \in[90,100[, p=2$ yielding $b \in[80,90[$, etc).

3) Tasks with equal benefit from 1) and 2) will be separated on a third hierarchy level, which consists of two parameters: First, the statistical probability enters as $\left(\xi_{H}-1\right)^{2}$ in order to rank rare opportunities (with low values of $\xi_{H}$ ) higher. Second, the user category enters as a configurable weighting factor which enables steering according to the mission's data policy. 


\section{Sunglint Avoidance Strategy}

\section{Motivation}

The specular reflection of sunlight from water surfaces is called sunglint. The amount of sunglint visible is a function of the viewing geometry between Sun and observer as well as the sea surface state [30]. However, even in case the water surface would be completely flat, sunglint does not only affect a single point in an observed scene due to the angular extension of the Sun. The influence of sea surface roughness on sunglint due to wind was first researched and described theoretically by Cox and Munk [31]: As the wind speed increases, the sunglint weakens close to the geometric center, whereas its intensity increases farther away.

Sunglint can have a blinding effect to the human eye as well as to a remote sensor in the optical and near infrared range: Water-leaving paths typically contribute about $15 \%$ to the total radiance [30]. Sunglint may multiply the received flux, consequently decrease the signal-to-noise ratio significantly and render it impossible to recreate useful data. In most cases, sunglint is thus a condition to be either avoided beforehand (e. g. [32]) or to be removed from images afterward through appropriate algorithms (see [30] for an overview). For EnMAP, it was decided to implement an avoidance strategy: With a similar motivation as for cloud handling (see Section III.B), this strategy aims at saving onboard resources by not scheduling acquisitions that are presumably highly useless for the researcher in favor of more promising scenes. A glint correction is currently not anticipated in the pre-processing of EnMAP images.

On the other hand, there also exist use cases where a researcher might want to explicitly aim at scenes with sunglint: As in the original work of Cox and Munk [31], one application is to study the sea surface state itself through the sunglint effect (e.g. [33]).

\section{Implementation}

The EnMAP user interface [34] will enable the researcher to choose which sunglint option best fits his research question:

1) No sunglint preference (default)

2) Explicitly avoid sunglint

3) Explicitly aim at sunglint

This selection is forwarded to MPS which evaluates each acquisition opportunity for sunglint (option 2 and 3 ) and subsequently excludes scenes which do not match the user requirement; this filter applies for the scene preview during preparation of the order as well as its final ingestion. In an advanced option, it can also be chosen how much of a scene may at most be affected by sunglint before it is considered useless or - in the contrary - how much of a scene should at least be affected by sunglint.

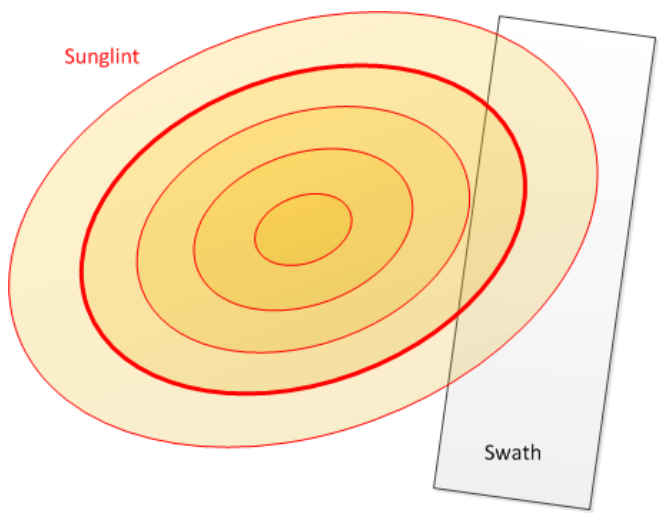

Fig. 2 Schematic illustration of a ground swath w.r.t. sunglint reflectance (increasing towards the central area where specular reflection would also be encountered for a flat sea surface). Red lines sketch positions of equal sunglint reflectance, and the thick red line indicates a reflectance threshold.

Whether a scene is affected by sunglint is assessed quantitatively with the MPS library SCOTA (Section III.A): Using a suitable ground sampling, it calculates the sunglint reflectance for each position of the swath (Fig. 2); regions with reflectances above a certain threshold are considered affected. If the affected area exceeds the user-specified limit w.r.t. the whole swath, the scene itself is considered affected. A schematic overview of the area affected by sunglint 
(corresponding to the intersection of thick red circle and swath in Fig. 2) can also be provided to the user during the order preview process on request.

The algorithm to calculate the sunglint reflectance itself was implemented following the description of [30]. The viewing geometry is known by the satellite orbit and the region of interest provided by the user. The algorithm also requires a probability distribution for the slopes of water facets, which can be approximated from wind speed; the baseline MPS implementation will use a simplified version of the Cox and Munk parametrization (first-order approximation, i. e. Gaussian). Since the user may enter an order well in advance where no reliable wind data is available (in particular for wind speeds close to the surface), the MPS calculates the sunglint reflectance for a predefined set of constant wind speeds and makes no distinction between down- and crosswind in the model. Without accurate knowledge of the wind speed, analyzing the reflectance values for more than one wind speed enables a conservative approach: In the avoidance option, this means that an area is considered affected if it exceeds the predefined threshold in any wind speed scenario.

\section{Verification}

The algorithm was verified and calibrated in cooperation with EOMAP For this purpose, 193 image areas from 103 Landsat [35] scenes from various regions and seasons have been analyzed in order to cover a wide range of geometric observing and sea surface conditions. Comparing results from a visual inspection with predictions using sunglint reflectances, this analysis showed that two wind speed scenarios (low and high) are sufficient to avoid or target at sunglint with a confidence level of $95 \%$. Furthermore, it yielded the corresponding reflectance threshold levels which will be used operationally for each sunglint option.

\section{Conclusion and Outlook}

Major novelties of the EnMAP MPS will be its high degree of responsiveness to user input and inclusion of additional information to estimate the feasibility of observation requests, which leads to new concepts compared to existing systems:

1) EnMAP shall be the first mission to benefit from the concept of the Reactive Planning framework [19], which particularly enables immediate user feedback and shorter order deadlines. In addition, via the EnMAP user interface [34], the user obtains detailed information regarding an observation request through a back end MPS swath preview service already before finally submitting the respective order.

2) The EnMAP MPS involves global cloud coverage information (predicted and historical) as well as estimated sunglint reflectance in the automatic process of scheduling observation requests for optical images of the Earth's surface. This way, the system is able to adapt the timeline to the users' goals: For most observation requests it helps to avoid wasting satellite and ground segment resources on images which contain little or no useful information. In other cases, clouds or sunglint areas themselves might be subject of interest; for such requests, clouds and/or sunglint may be a prerequisite for execution.

Being a first application of these concepts, the EnMAP MPS comprises not all of their potential features. However, the functionality might gradually be extended as the experience with the system grows and the mission evolves with respect to Mission Planning requirements; examples include:

- Dynamic ground station scenario: Uplink and downlink contacts may be ingested on short notice, including adaptation of the uplink schedule.

- Robustness against uplink failures: Alternative timelines and the respective commands are prepared in advance, covering cases where an uplink partly fails. The TimelineManager may prepare alternative timelines to command and the respective commands, e. g. for scenarios where in-pass modifications of the timeline are required, see [36].

- Order preview with manual conflict resolution: This feature consists of a preliminary planning run, which informs the authorized operator about possible consequences of sending an order, e. g. which other orders need to be moved or discarded in order to allow the new order to be planned. In case multiple alternative consequences exist, these alternatives may be presented and the operator may select his preferred solution.

The integration and operation phases will also enable a verification and optimization of system parameters, e.g.:

- Prioritization: Monitoring the actual dynamics of the planning process will enable a deeper understanding for the subtle interaction of cloud forecasts, user priorities, contingents, and timeline stability. Regular reviews of the involved parameters may optimize the mission's performance in general and with respect to its policy.

\footnotetext{
†https://www.eomap.com
} 
- Cloud coverage strategy validation: As the observational database grows, the chosen approach for cloud forecast can be validated against reality, and the involved parameters (e. g. forecast horizon) will be optimized.

- Sunglint verification: Initial wind speeds and thresholds for sunglint reflectance have been determined using Landsat scenes; repeating this analysis using EnMAP data would yield specifically adapted parameters as well as an additional verification of the approach.

\section{Acknowledgements}

Supported by the DLR Space Administration with funds of the German Federal Ministry of Economic Affairs and Technology on the basis of a decision by the German Bundestag (50 EE 0850).

\section{References}

[1] Knipling, E. B., "Physical and physiological basis for the reflectance of visible and near-infrared radiation from vegetation," Remote Sensing of Environment, Vol. 1, No. 3, 1970, pp. 155-159. doi:10.1016/S0034-4257(70)80021-9, URL https: //doi.org/10.1016/S0034-4257(70)80021-9

[2] Lee, Z., Carder, K. L., Mobley, C. D., Steward, R. G., and Patch, J. S., "Hyperspectral remote sensing for shallow waters: 2. Deriving bottom depths and water properties by optimization," Appl. Opt., Vol. 38, No. 18, 1999, pp. 3831-3843. doi:10.1364/AO.38.003831, URL http://ao.osa.org/abstract.cfm?URI=ao-38-18-3831.

[3] Ben-Dor, E., Chabrillat, S., Demattê, J. A., Taylor, G., Hill, J., Whiting, M., and Sommer, S., "Using Imaging Spectroscopy to study soil properties," Remote Sensing of Environment, Vol. 113, 2009, pp. S38-S55. URL https://www.researchgate. net/publication/223042783_Using_Imaging_Spectroscopy_to_study_soil_properties

[4] Hunt, G. R., "Spectral signatures of particulate minerals in the visible and near infrared," Geophysics, Vol. 42, No. 3, 1977, pp. 501-513. doi:10.1190/1.1440721, URL http://dx.doi.org/10.1190/1.1440721

[5] Dozier, J., Green, R. O., Nolin, A. W., and Painter, T. H., "Interpretation of snow properties from imaging spectrometry," Remote Sensing of Environment, Vol. 113, 2009, pp. S25-S37. doi:10.1016/j.rse.2007.07.029, URL https://doi.org/10. $1016 /$ j.rse.2007.07.029

[6] Heldens, W., Heiden, U., Esch, T., Stein, E., and Müller, A., "Can the Future EnMAP Mission Contribute to Urban Applications? A Literature Survey," Remote Sensing, Vol. 3, No. 9, 2011, pp. 1817-1846. URL http://elib.dlr.de/70793/

[7] Guanter, L., Kaufmann, H., Segl, K., Foerster, S., Rogass, C., Chabrillat, S., Kuester, T., Hollstein, A., Rossner, G., Chlebek, C., Straif, C., Fischer, S., Schrader, S., Storch, T., Heiden, U., Müller, A., Bachmann, M., Mühle, H., Müller, R., Habermeyer, M., Ohndorf, A., Hill, J., Buddenbaum, H., Hostert, P., van der Linden, S., Leitão, P. J., Rabe, A., Doerffer, R., Krasemann, H., Xi, H., Mauser, W., Hank, T., Locherer, M., Rast, M., Staenz, K., and Sang, B., "The EnMAP Spaceborne Imaging Spectroscopy Mission for Earth Observation,” Remote Sensing, Vol. 7, No. 7, 2015, pp. 8830-8857. doi:10.3390/rs70708830, URL http://www.mdpi.com/2072-4292/7/7/8830

[8] Sang, B., Schubert, J., Kaiser, S., Mogulsky, V., Neumann, C., Förster, K.-P., Hofer, S., Stuffler, T., Kaufmann, H., Müller, A., Eversberg, T., and Chlebek, C., "The EnMAP hyperspectral imaging spectrometer: instrument concept, calibration, and technologies," Proc. SPIE, Vol. 7086, 2008, pp. 708605 1-15. doi:10.1117/12.794870, URL http://dx . doi . org/10.1117/ 12.794870

[9] Guanter, L., Kaufmann, H., Foerster, S., Brosinsky, A., Wulf, H., Bochow, M., Boesche, N., Brell, M., Buddenbaum, H., Chabrillat, S., Hank, T., Heiden, U., Heim, B., Heldens, W., Hill, J., Hollstein, A., Hostert, P., Krasemann, H., Leitão, P. J., van der Linden, S., Mauser, W., Mielke, C., Müller, A., Oppelt, N., Roessner, S., Röttgers, R., Schneiderhan, T., Staenz, K., and Segl, K., "EnMAP Science Plan,” Tech. rep., GFZ Data Services, 2016. doi:10.2312/enmap.2016.006, URL http://doi.org/10.2312/enmap.2016.006

[10] Storch, T., Eberle, S., Makasy, C., Maslin, S., de, A. M., Mißling, K.-D., Mühle, H., Müller, R., Engelbrecht, S., Gredel, J., and Müller, A., "On the Design of the Ground Segment for the Future Hyperspectral Satellite Mission EnMAP," 2010 IEEE Aerospace Conference, IEEE, 2010, pp. 1-11. URL http://elib.dlr.de/59778/

[11] Storch, T., Habermeyer, M., Eberle, S., Mühle, H., and Müller, R., "Towards a Critical Design of an Operational Ground Segment for an Earth Observation Mission,” Journal of Applied Remote Sensing, Vol. 7, No. 1, 2013, pp. 1-12. URL http://elib.dlr.de/81601/ 
[12] Habermeyer, M., Storch, T., Eberle, S., Makasy, C., Maslin, S., de Miguel, A., Missling, K.-D., Mühle, H., Müller, R., Engelbrecht, S., Gredel, J., and Heiden, U., "Ground Segment Design of the EnMAP Hyperspectral Satellite Mission," Hyperspectral Workshop 2010, Vol. SP-683, edited by H. Lacoste-Francis, ESA Communications, 2010. doi:10.2514/6.2016-2568, URL http://elib.dlr.de/66561/

[13] Müller, R., Bachmann, M., Miguel, A., Müller, A., Neumann, A., Palubinskas, G., Richter, R., Schneider, M., Storch, T., Walzel, T., Kaufmann, H., Guanter, L., Segl, K., Heege, T., and Kiselev, V., "The Processing Chain and Cal/Val Operations of the Future Hyperspectral Satellite Mission EnMAP,” 2010 IEEE Aerospace Conference, 2010, pp. 1-9. URL http://elib.dlr.de/63948/

[14] Missling, K.-D., Damerow, H., Habermeyer, M., Kaufmann, H., Maass, H., Mühle, H., Müller, R., Schwarz, J., Storch, T., Tegler, M., and Tian, T., "Payload Ground Segment of the EnMAP Mission," Proceedings of the 45th Ziolkovski Conference, edited by M. Marov, Russian Academy of Sciences, Kaluga, Russia, 2011, pp. 23-29. URL/http://elib.dlr.de/71229/

[15] Habermeyer, M., Storch, T., Eberle, S., Makasy, C., de Miguel, A., Missling, K.-D., Mühle, H., and Müller, R., "Design of the Integration and Technical Verification and Validation Phase of the Ground Segment of the Hyperspectral Satellite Mission EnMAP,” EARSeL SIG-IS Workshop 2011, 2011. URL http://elib.dlr.de/72108/

[16] Maurer, E., Mrowka, F., Braun, A., Geyer, M. P., Lenzen, C., Wasser, Y., and Wickler, M., “TerraSAR-X Mission Planning System: Automated Command Generation for Spacecraft Operations," IEEE Transactions on Geoscience and Remote Sensing, Vol. 48, No. 2, 2010, pp. 642-648. URL http://elib.dlr.de/63512/

[17] Axmann, R., and Eberle, S., "Mission Planning and Operational Constraints and Their Resolution for EO Missions Like EnMAP," SpaceOps 2010 Conference, American Institute of Aeronautics and Astronautics, Huntsville, Alabama, USA, 2010. URL https: //doi .org/10.2514/6.2010-2218.

[18] Hartung, J., Nibler, R., Spörl, A., Lenzen, C., Wörle, M. T., and Peat, C., "GSOC SoE-Editor 2.0 - A Generic Sequence of Events Tool," 14th International Conference on Space Operations, Daejeon, Republic of Korea, 2016. URL http: //elib.dlr.de/105083/

[19] Wörle, M. T., Lenzen, C., Göttfert, T., Spörl, A., Grishechkin, B., Mrowka, F., and Wickler, M., "The Incremental Planning System - GSOC's Next Generation Mission Planning Framework," 13th International Conference on Space Operations, Pasadena, California, USA, 2014. URL http://elib.dlr.de/89586/

[20] Mrowka, F., Geyer, M. P., Lenzen, C., Spörl, A., Göttfert, T., Maurer, E., Wickler, M., and Schättler, B., “The Joint TerraSAR-X / TanDEM-X Mission Planning System,” Symposium Proceedings of IGARSS 2011, Vancouver, Canada, 2011, pp. 3971-3974. URL http://elib.dlr.de/74917

[21] Mrowka, F., Göttfert, T., Wörle, M. T., Schättler, B., and Stathopoulos, F., “The TerraSAR-X/TanDEM-X Mission Planning System: Realizing new Customer Visions by Applying new Upgrade Strategies," 14th International Conference on Space Operations, Deajeon, Republic of Korea, 2016. URL http://elib.dlr.de/104596.

[22] Gasch, J. R., and Campana, K. A., "Cloud cover avoidance in space-based remote sensing acquisition," Algorithms for Multispectral, Hyperspectral, and Ultraspectral Imagery VI, Proc. SPIE, Vol. 4049, edited by S. S. Shen and M. R. Descour, 2000, pp. 336-347. doi:10.1117/12.410357, URL https://doi.org/10.1117/12.410357

[23] Axmann, R., "Interactive Acquisition Scheduling for Low Earth Orbiting Satellites," Diss., Technische Universität München, 2010. URL http://mediatum.ub.tum.de/doc/823513

[24] Fletcher, P. A., "Image acquisition planning for the CHRIS sensor onboard PROBA," Imaging Spectrometry X, Vol. 5546, edited by S. S. Shen and P. E. Lewis, 2004, pp. 141-148. doi:10.1117/12.561097, URL https://doi .org/10.1117/12.561097.

[25] Grishechkin, B., Braun, A., and Wickler, M., "Optimization of Positioning of Ground Stations for Space Optical Missions," 12th International Conference on Space Operations, Stockholm, Sweden, 2012. URL http://elib.dlr.de/77055/

[26] Stengel, M., Stapelberg, S., Sus, O., Schlundt, C., Poulsen, C., Thomas, G., Christensen, M., Carbajal Henken, C., Preusker, R., Fischer, J., Devasthale, A., Willén, U., Karlsson, K.-G., McGarragh, G. R., Proud, S., Povey, A. C., Grainger, R. G., Meirink, J. F., Feofilov, A., Bennartz, R., Bojanowski, J. S., and Hollmann, R., "Cloud property datasets retrieved from AVHRR, MODIS, AATSR and MERIS in the framework of the Cloud_cci project," Earth System Science Data, Vol. 9, No. 2, 2017, pp. 881-904. doi:10.5194/essd-9-881-2017, URL https://www . earth-syst-sci-data.net/9/881/2017/ 
[27] Stengel, M., Sus, O., Stapelberg, S., Schlundt, C., Poulsen, C., and Hollmann, R., "ESA Cloud_cci cloud property datasets retrieved from passive satellite sensors: AVHRR-PM L3C/L3U cloud products - Version 2.0," Deutscher Wetterdienst (DWD), 2017. doi:10.5676/DWD/ESA_Cloud_cci/AVHRR-PM/V002, URL https://doi.org/10.5676/DWD/ESA_Cloud_cci/ AVHRR-PM/VQQ2

[28] Reinert, D., Prill, F., Frank, H., Denhard, M., and Zängl, G., Database Reference Manual for ICON and ICON-EPS (Version 1.2.2, Deutscher Wetterdienst, 2018. doi:10.5676/DWD_pub/nwv/icon_1.2.2, URL/https://d-nb.info/1081305452/34

[29] Grishechkin, B., Braun, A., and Wickler, M., "Clouds Handling for Planning of Optical Space Missions," 12th International Conference on Space Operations, Stockholm, Sweden, 2012. URL http://elib.dlr.de/77056/.

[30] Kay, S., Hedley, J. D., and Lavender, S., "Sun Glint Correction of High and Low Spatial Resolution Images of Aquatic Scenes: a Review of Methods for Visible and Near-Infrared Wavelengths," Remote Sensing, Vol. 1, No. 4, 2009 , pp. 697-730. doi:10.3390/rs1040697, URL http://www .mdpi .com/2072-4292/1/4/697.

[31] Cox, C., and Munk, W., "Measurement of the Roughness of the Sea Surface from Photographs of the Sun's Glitter," J. Opt. Soc. Am., Vol. 44, No. 11, 1954, pp. 838-850. doi:10.1364/JOSA.44.000838, URL https://doi.org/10.1364/J0SA.44. 000838.

[32] Mailhe, L. M., Schiff, C., and Stadler, J. H., "Calipso's Mission Design: Sun-Glint Avoidance Strategies," Advances in Astronautical Sciences, Vol. 119, 2005, pp. 195-213. URL https://ntrs.nasa.gov/search.jsp?R=20040081137

[33] Ebuchi, N., and Kizu, S., "Probability Distribution of Surface Wave Slope Derived Using Sun Glitter Images from Geostationary Meteorological Satellite and Surface Vector Winds from Scatterometers," Journal of Oceanography, Vol. 58, No. 3, 2002, pp. 477-486. doi:10.1023/A:1021213331788, URL http://dx.doi.org/10.1023/A\%3A1021213331788

[34] Heiden, U., Pinnel, N., Mühle, H., Pengler, I., and Storch, T., “The EnMAP user interface and user request scenarios," Proceedings of the EARSeL 7th SIG-Imaging Spectroscopy Workshop, Edinburgh, United Kingdom, 2011, pp. 1-6. URL http://elib.dlr.de/72267/

[35] Wulder, M. A., White, J. C., Loveland, T. R., Woodcock, C. E., Belward, A. S., Cohen, W. B., Fosnight, E. A., Shaw, J., Masek, J. G., and Roy, D. P., "The global Landsat archive: Status, consolidation, and direction," Remote Sensing of Environment, Vol. 185, 2016, pp. 271-283. doi:https://doi.org/10.1016/j.rse.2015.11.032, URL http://www.sciencedirect . com/science/ article/pii/S0034425715302194

[36] Göttfert, T., Lenzen, C., Wörle, M. T., Mrowka, F., and Wickler, M., "Robust Commanding," 13th International Conference on Space Operations, Pasadena, California, USA, 2014. URL http://elib.dlr.de/89617/ 